\title{
Transatlantica
}

Revue d'études américaines. American Studies Journal

\section{Quand le savant l'emporte sur le politique}

The Declining Significance of Race de William Julius Wilson

\section{David Diallo}

\section{(2) OpenEdition}

Journals

Édition électronique

URL : https://journals.openedition.org/transatlantica/4361

DOI : 10.4000/transatlantica.4361

ISSN : $1765-2766$

Éditeur

Association française d'Etudes Américaines (AFEA)

Référence électronique

David Diallo, «Quand le savant l'emporte sur le politique », Transatlantica [En ligne], 1 | 2009, mis en ligne le 03 juillet 2009, consulté le 16 septembre 2021. URL : http://journals.openedition.org/

transatlantica/4361; DOI : https://doi.org/10.4000/transatlantica.4361

Ce document a été généré automatiquement le 16 septembre 2021.

\section{cc)}

Transatlantica - Revue d'études américaines est mise à disposition selon les termes de la licence Creative Commons Attribution - Pas d'Utilisation Commerciale - Pas de Modification 4.0 International. 


\title{
Quand le savant l'emporte sur le politique
}

\author{
The Declining Significance of Race de William Julius Wilson
}

\section{David Diallo}
«Le savant doit refouler les sentiments qui le lient à l'objet, les jugements de valeur qui surgissent spontanément en lui et commandent son attitude à l'égard de la société, celle d'hier qu'il explore et celle d'aujourd'hui qu'il désire, quoi qu'il en ait, sauvegarder, détruire ou
changer"
Raymond Aron (Max Weber, Le Savant et le
Politique $)^{1}$

1 The Declining Significance of Race: Blacks and Changing American Institutions, l'ouvrage du sociologue américain William Julius Wilson publié en 1978 constitue un tournant dans les sciences sociales américaines. Le livre, en raison de la thèse qu'il soutient, que les opportunités professionnelles des Noirs étaient, à la fin des années soixante-dix et dans le secteur économique, davantage déterminées par un facteur social que racial, provoqua une vive controverse. Dans un entretien avec l'auteur diffusé sur la chaîne PBS en 1997, l'universitaire Henri Louis Gates Jr. évoque la polémique que suscita l'ouvrage de son confrère en ces termes:

In 1978 you published The Declining Significance of Race. People lined up from here to China within the black community to be upset about that title. People who hadn't even read the book, because they didn't want race to be in decline as a significant variable in their oppression"

2 En dépit de sa rigueur méthodologique, récompensée par ses pairs de l'American Sociological Association qui lui attribuèrent le Sydney M. Spivack Award, l'ouvrage de Wilson provoqua de vives réactions parmi ses confrères et chez de nombreux membres de l'intelligentsia noire. En effet, en présentant un travail explicatif dégagé d'enjeux idéologiques dans un domaine de recherche qui recèle de nombreux travaux partisans et autres ouvrages universitaires à vocation militante, Wilson confondit un grand 
nombre d'intellectuels et d'activistes pour lesquels réduire l'importance du facteur racial, quelle que soit la méthode ou sa validité scientifique, s'apparentait à un acte de déloyauté et de désolidarisation envers sa communauté d'origine.

Cet article à pour ambition, à travers l'étude des réactions suscitées par cet ouvrage dans le monde universitaire, d'examiner le clivage fort qui existe dans le domaine de la recherche sur les Noirs Américains entre partisans d'un discours essentialiste et particulariste sur la condition des Noirs aux Etats-Unis et les tenants d'une approche plus sociologique. Je m'arrêterai en premier lieu sur une des réactions les plus virulentes auxquelles The Declining Significance of Race donna lieu pour en faire l'analyse. J'examinerai ensuite les enjeux symboliques autour de cette réaction, plus émotionnelle que rationnelle comme je tâcherai de le démontrer, pour aborder la question sousjacente mais néanmoins capitale du rapport à l'objet dans les travaux universitaires sur les Noirs Américains.

4 The Declining Significance of Race, sur lequel de nombreux chercheurs se sont déjà penchés, a été commenté à maintes reprises, aussi je m'arrêterai que très brièvement sur son contenu, largement couvert à mon sens ${ }^{3}$. Retenons principalement que William Julius Wilson, au terme d'un travail de recherche méticuleux s'appuyant sur des analyses de données, de rapports gouvernementaux, et d'ouvrages universitaires, y affirme que les changements politiques qui suivirent la Seconde Guerre mondiale et les changements structurels de l'économie contribuèrent conjointement à un processus graduel de déracialisation dans le secteur économique. Selon lui, ces changements réduirent progressivement l'importance de la race comme facteur déterminant dans la mobilité socioéconomique des Noirs aux Etats-Unis. Cette thèse soutient notamment que l'expansion du secteur économique participa au déplacement des Noirs du sud rural vers les centres industriels du nord et contribua à une création d'emplois qui donna lieu à une plus grande diversification professionnelle au sein de la population noire. Par ailleurs, Wilson estime que l'Etat, suite au mouvement en faveur des droits civiques qui eut lieu dans les années 1960, au lieu de renforcer les barrières raciales mises en place lors des siècles précédents, a, dans les années précédant l'ouvrage, oeuvré pour la promotion d'une égalité raciale. Comme il le remarque, la combinaison de changements économiques et politiques de cette époque entraîna une plus grande mobilité économique et procura plus d'occasions à une partie considérable de la population noire. Wilson n'omet toutefois pas de préciser qu'alors que la croissance économique qui suivit la Deuxième Guerre Mondiale permit à de nombreux Noirs d'améliorer leur situation, des transformations plus récentes de l'économie du pays ont quant à elles contribué à la détérioration de la couche la plus défavorisée de la population noire alors même que des programmes et lois anti-discrimination sans précédents étaient mis en place.

5 Il est important d'indiquer que lorsqu'il avança dans son ouvrage que le facteur racial n'était plus un élément déterminant, Wilson n'affirmait guère, comme une lecture hâtive de son ouvrage qui s'arrêterait à son titre pourrait le laisser penser, que le racisme aux Etats-Unis était sur le déclin. Le sociologue tentait simplement de démontrer comment, au début des années 1960 et pour la première fois dans l'histoire des Noirs Américains, une classe moyenne parvenait à transmettre à ses enfants les différentes formes de capital nécessaires pour conserver, voire améliorer, comme les Blancs l'avaient fait jusque là, leur statut de classe. Selon lui, l'appartenance à une 
classe était devenue plus importante que la race d'un individu noir pour déterminer ses chances.

6 Si cette vision d'une cristallisation d'une structure de classe au sein de la population noire est aujourd'hui communément acceptée chez les chercheurs en sciences sociales et que l'écart grandissant entre une classe moyenne noire et une classe noire largement défavorisée n'est plus débattu, il n'en était pas de même, comme nous pourrons le constater, au moment de la publication du livre de Wilson.

7 Dès sa parution, cet ouvrage mit une partie de la communauté scientifique en émoi et suscita des réactions virulentes chez plusieurs intellectuels et leaders de la communauté noire pour lesquels remettre ainsi en question l'importance du facteur racial, quelle que soit la méthode ou sa validité scientifique, fut perçu comme une véritable désolidarisation d'avec la communauté noire. Si, comme le rappelle d'ailleurs l'essayiste Gerald Early dans un entretien avec Wilson, ce dernier déclencha une tempête de protestations lorsqu'il avança que la classe et l'économie jouaient un rôle plus déterminant que la race dans la situation difficile des résidents noirs des grands centres urbains ${ }^{4}$, rares furent celles qui dépassèrent celle du Professeur Alphonso Pinkney, qui, en 1984, publia un ouvrage antagonique intitulé The Myth of Black Progress. Dans ce livre, ce sociologue répondait directement à Wilson et à d'autres chercheurs néo-conservateurs «aux intentions mystificatrices » auxquels il l'associait et dont les travaux, d'après lui, influençaient fortement les politiques publiques de l'époque ${ }^{5}$.

Dans son livre, Pinkney s'en prenait particulièrement aux récents ouvrages rédigés par des chercheurs en sciences sociales, noirs et blancs, qui ne considéraient plus la race comme une variable fondamentale dans l'analyse des relations entre noirs et blancs et dont les travaux, comme il l'affirmait dans son introduction (plus particulièrement dans un chapitre intitule Social Scientists and the Myth of Black Progress), apportaient des arguments aux conservateurs au pouvoir. Pinkney envisageait donc, dans cet ouvrage, de déconstruire leurs arguments et de dévoiler le mythe du progrès des Noirs dans la société américaine qu'ils véhiculaient ${ }^{6}$. S'il partageait l'avis des auteurs dont il remettait le travail en question sur certains points, et concédait que le racisme était certainement moins prononcé aux Etats-Unis depuis la période de lutte pour les droits civiques et le Civil Rights Act of 1964, Pinkney estimait néanmoins que la race continuait, à l'époque de la parution des travaux critiqués, de dominer les rapports sociaux et économiques aux Etats-Unis. Il s'opposait notamment aux idées de Wilson, qui l'inquiétaient d'autant plus qu'il s'agissait de celles d'un chercheur noir. Comme il l'explique au début de son livre:

There are so many faulty (even naive) interpretations in this short book that it is an amazing piece of work coming from a black sociologist. In the first place there is evidence that the economic gap between black and white workers is widening rather than narrowing, and discrimination against blacks, regardless of class, continues to be widespread.?

Pour Pinkney, les propos de Wilson s'appuyaient non seulement sur des commentaires peu convaincants, mais étaient littéralement choquants "pour la plupart des Noirs américains $»^{8}$. Pinkney termine d'ailleurs sa recension du livre de Wilson en s'arrêtant longuement sur les critiques formulées à l'encontre de ce dernier par l'Association of Black Sociologists. En septembre 1978, lors de sa réunion annuelle, ses membres y exprimèrent leur indignation sur ce qu'ils considéraient être une représentation erronée de l'expérience noire. Ils estimaient notamment que l'attention portée à l'ouvrage de Wilson obscurcissait considérablement le problème de l'oppression 
persistante que subissait les Noirs Américains, en dépit d'un grand nombre de rapports récents, comme celui du Department of Housing and Urban Development, dont les résultats contredisaient, à leurs yeux, la thèse soutenue par Wilson. Pinkney rapporte quelques unes de leurs remarques pour étayer son point de vue :

The book clearly omits significant data regarding the continuing discrimination against blacks at all class levels. It misinterprets even facts presented in the volume, and draws inferences that are contrary to the conclusions that other black and white scholars have reached with reference to the salience of race as a critical variable in American society (...)

In the past reactionary groups have seized upon inappropriate analyses as a basis to the further suppression of blacks. We would hope that this is not the intent of the recent recognition that has been given to Professor Wilson's book... We are also extremely disturbed over the policy implications that may derive from his work and that, given the nature of American society, are likely to set in motion equally objectionable trends in funding, research, and training. ${ }^{9}$

A plusieurs reprises dans son ouvrage, Pinkney reviendra sur les thèses de Wilson, qu'il qualifiera sans détours de polémiques et troublantes ${ }^{10}$. S'il reconnaissait la véracité de certains points traités par Wilson et admettait l'idée de la structuration de la population noire en classes, Pinkney estimait néanmoins que les conclusions de Wilson étaient, à son avis, bien trop prématurées pour parler de tendance.

11 Hormis leurs divergences d'interprétations des chiffres du Bureau of the Census ou du Department of Labor ${ }^{11}$, les reproches faits par Pinkney à Wilson révélaient surtout le net parti pris idéologique du premier. En effet, le positionnement politique de Pinkney sur la question raciale transparaissait nettement, aussi bien dans la forme de ses remarques que dans leur fond. Au regard des critiques que Pinkney formula à l'encontre du travail (et de la personne) de William Julius Wilson, ce n'était guère son approche méthodologique, extrêmement rigoureuse et répondant aux critères de scientificité généralement attendus de travaux en sciences sociales, mais la nature même de son entreprise qui lui étaient reprochée.

12 Comme il l'expliqua ultérieurement avec soin, William Julius Wilson, dont la démarche sociologique reflète le précepte spinoziste «ne pas déplorer, ne pas rire, ne pas détester mais comprendre ", entreprenait, dans The Declining Significance of Race, de faire l'analyse de facteurs trop souvent relégués aux arrière-plans alors qu'ils avaient, à ses yeux, un impact significatif sur la population noire des Etats-Unis ${ }^{12}$. En rendant saillant ces facteurs extra-raciaux, le sociologue ne faisait d'ailleurs que poursuivre le travail entrepris par des chercheurs comme l'économiste Vivian Henderson qui avait déjà publié plusieurs ouvrages sur la dimension économique dans les relations raciales et sur le statut économique des Noirs ${ }^{13}$. Ce dernier avait en effet souligné, explicitement et bien avant Wilson, dans quelle mesure les changements technologiques et économiques avaient commencé à reléguer la race au second plan ${ }^{14}$.

Comme Wilson l'expliqua ultérieurement et plus en détail dans un article intitulé « The Black Community: Race and Class ", de nombreux développements récents dans la communauté noire n'avaient pas été, selon lui, suffisamment pris en compte (Wilson 1994). Il y remarquait, en revanche, qu'en dépit de signaux objectifs d'une amélioration des conditions économiques pour une partie de la population noire, un flot incessant de rapports pessimistes sur la montée en flèche des chiffres du chômage chez les Noirs, sur leur participation décroissante à la force de travail, sur l'augmentation considérable du nombre de Noirs bénéficiant de l'aide sociale, ou encore sur l'explosion 
du nombre de familles tenues par une mère seule (sans oublier les problèmes perpétuels de criminalité) venait entretenir un sentiment de démoralisation dans la communauté noire et chez ses élites. Sentiment qui donnait par ailleurs du grain à moudre à des intellectuels guidés par un chauvinisme racial et dont les travaux partisans étaient régulièrement repris par les activistes et leaders politiques Noirs. Or, comme Wilson le montra dans The Declining Significance of Race, une analyse minutieuse des problèmes de la population noire aux Etats-Unis révélait que des variations considérables n'avaient pas été notées et appréciées, et que les effets de programmes et de politiques mis en place sur différents segments de la population avaient rarement été pris en compte. D'où sa volonté d'examiner des facteurs qui non seulement avaient des implications profondes sur la structure de la communauté noire mais qui, par ailleurs, n'avaient pas reçu suffisamment d'attention ou avaient été systématiquement rejetés par des intellectuels militants dont les travaux exaltaient davantage une idéologie raciale destinée à fomenter un fort sentiment de solidarité chez les Noirs. Wilson analyse finement cette démarche, regrettable à ses yeux dans la mesure où l'objectivité scientifique de ses adeptes laisse place à un sentimentalisme partisan, en ces termes :

However, arguments stressing economic relations in determining the structure of inequality and in significantly influencing the experiences of inequality began to compete with a new definition, description, and explanation of the black condition. This new approach, proclaimed as a "black perspective", revealed an ideological shift from interracialism to racial solidarity. It first gained currency among militant black spokesmen in the late 1960s and became a theme in the writings of young black academics and intellectuals by the early 1970s. Although the "black perspective" represented a variety of views and arguments on issues of race, the trumpeting of racial pride and self-affirmation was common to all the writings and speeches on the subject. ${ }^{15}$

15 A ce titre, l'ouvrage de Wilson marque clairement sa différence avec cette littérature partisane qui mettait principalement l'accent sur le facteur racial et qui n'attribuait que peu d'attention aux autres éléments déterminants. Une telle attitude, comme le souligna d'ailleurs Wilson, fit que plus d'attention fut par exemple accordée à l'affaire Bakke qu'au Humphry/ Hawkins full employement bill qui, selon lui, était bien plus important pour remédier aux problèmes des travailleurs noirs et des pauvres ${ }^{16}$. Or, la focalisation mystificatrice sur le facteur racial des tenants d'un discours essentialiste et militant détournait l'attention de pareilles solutions aux problèmes économiques de la population noire, dont les difficultés étaient, comme le soulignait Wilson, étroitement liées à la structure de l'économie américaine et au marché du travail.

$\mathrm{Si}$, pour reprendre les mots de Max Weber, la posture de Wilson dans The Declining Significance of Race est incontestablement celle du "savant ", celle des défenseurs d'une «black perspective » - illustrée ici par le discours idéologiquement motivé de Pinkney , ne répond guère à ses critères. Weber, dans Le Savant et le Politique soulignait à plusieurs reprises que l'idéologie politique n'avait rien à faire dans l'université et répète que les vertus du politique étaient incompatibles avec celle du savant. Comme Raymond Aron le résume dans son introduction de l'édition de 1991:

On ne peut pas être en même temps homme d'action et homme d'études, sans porter atteinte à la dignité de l'un ou l'autre métier, sans manquer à la vocation de l'un ou de l'autre ${ }^{17}$

17 Toutefois, si pour Weber la fin ultime de la pensée peut être la participation à l'action ${ }^{18}$, celui-ci estimait qu'une prise de position était néanmoins possible en dehors de 
l'université et du monde de la recherche dans la mesure ou la possession d'un savoir objectif pouvait être favorable à une action raisonnable.

D'ailleurs, les points de vue et les arguments de Wilson et de Pinkney divergent précisément sur le terrain de l'objectivité. Alors que le travail de Wilson répond aux critères d'exigence de la recherche en sciences sociales (définition des termes, présentation d'un cadre théorique, construction de l'objet...), la pensée de Pinkney laisse en revanche transparaître des prises de positions idéologiques très marquées. Une remarque comme, par exemple, celle où il regrette qu'un sociologue noir puisse tenir de tels propos (" it is an amazing piece of work coming from a black sociologist ») est manifestement le produit d'un jugement de valeur formulé sous le couvert de l'analyse. Tout comme celle bien plus âpre, qu'il exprime à la fin de son introduction :

Black social scientists, as well as white, appear to be supporting the growing conservative movement in the U.S. That white social scientists should engage in these activities is not surprising. However, black sociologists who support the conservative movement are not unlike government officials in (formerly) South Vietnam who supported American aggression against their own people. ${ }^{19}$

Pinkney pose ici, avec un sous-entendu raciste qui dévoile un chauvinisme racial motivé par une logique simplificatrice du type « eux » contre «nous », la question de la légitimité d'écrire sur les problèmes des Noirs dans un milieu où l'absence de parti pris est, théoriquement, un pré requis à tout travail de recherche. Il reproche par ailleurs à Wilson son absence de sensibilité raciale au profit d'une posture scientifique qu'ils devraient tous deux, en principe, partager, et dont l'objectif est de contribuer à une œuvre de clarté.

Alors que Wilson, dans The Declining Significance of Race refoulait soigneusement les sentiments qui pouvaient le lier à son objet ${ }^{20}$, les accents idéologiques et l'emportement qui caractérisent les propos de Pinkney permettent de justifier son attitude à l'égard du travail de Wilson. Ce dernier, dans The Myth of Black Progress, manifestait un parti pris idéologique pratique là où Wilson, dans The Declining Significance of Race, analysait scientifiquement des structures politiques et économiques pour mesurer leur impact sur la population noire. De ce fait et à la lumière des écrits de Max Weber, les arguments et les mots forts de Pinkney ressemblent plus à un appel politique destiné à susciter des prises de position chez les lecteurs que les moyens d'une analyse scientifique $^{21}$.

21 Le débat discursif qui opposait Pinkney à Wilson illustre précisément l'opposition, évoquée précédemment, d'un tenant d'une posture intellectuelle militante et partisane à un chercheur dont la vocation première était d'établir une vérité scientifique qui, bien qu'elle parût intolérable à son détracteur, répondait à l'objectivité des sciences sociales. Alors que l'argumentation de William Julius Wilson reposait sur un ensemble de données objectives et se limitait à un registre scientifique, celle d'Alfonso Pinkney, en revanche, allait régulièrement puiser ses justifications dans le registre émotionnel. Dès les premières lignes de son ouvrage par exemple, Pinkney privilégie l'anecdote aux résultats statistiques :

Reports of the ongoing oppression of Afro-Americans are commonplace: in 1983 a five-year-old black child was murdered in California by a white police officer who claimed that the child had a handgun; four blacks were indiscriminately killed by New Orleans police following the murder of a white police officer; a black transit worker was killed by a white mob in Brooklyn as he stopped with co-workers in a delicatessen for food; white voters refused to support well-qualified black 
candidates, the black candidate for mayor of Chicago, along with a democratic candidate for president, was refused permission to speak by a crowd of white racists at a Roman Catholic church on Palm Sunday; spokespersons for the administration continued to take position opposed to the aspirations blacks; twice as many black adults were unemployed as whites and among teenagers it was three blacks for every white; Little Rock desegregated its schools because of white boycotts. $^{22}$ avant et les présentant comme des vecteurs de légitimation s'adressant aux émotions plutôt qu'aux jugements rationnels Pinkney cherchait évidemment à le convaincre de la persistance du racisme aux Etats-Unis et à invalider, bien qu'elle soit ici caricaturée, dénaturée, et sortie de son contexte exclusivement économique, la thèse de Wilson. Alors que Wilson, recourant à des principes méthodologiques éprouvés, cherchait à écarter ses lecteurs des prénotions tenaces sur la situation économique des Noirs, Pinkney, contournant les facultés rationnelles de ses lecteurs, nourrissait leurs intuitions premières à coup d'anecdotes émouvantes en dépit des conditions d'utilisation des principes méthodologiques de la sociologie. ${ }^{23}$ sont assez fréquents dans les sciences sociales, plus particulièrement dans les travaux sur les Noirs Américains où le lecteur doit souvent discerner les vérités scientifiques des vérités intuitives. Une myopie analytique comparable à celle attribuée par Wilson aux tenants d'un discours partisan et idéologiquement motivé fut, par exemple, responsable de l'omission de tout un ensemble de facteurs importants dans les études sur la musique rap.

En effet, le lien originel entre le mouvement hip-hop et les pratiques socioculturelles des gangs de jeunes, s'il est généralement reconnu par les chercheurs qui se sont intéressés à la musique rap, a régulièrement été occulté par de multiples récits légitimateurs teintés de fierté raciale ${ }^{24}$. Les origines semi criminelles du rap, susceptibles d'enrayer la légitimation de cette musique comme pratique artistique, sont, tout comme la thèse de Wilson qui soutenait que la classe et l'économie jouaient un rôle plus déterminant que la race dans la situation difficile des résidents noirs des grands centres urbains à la fin des années 70 , souvent mentionnées laconiquement, comme si elles constituaient un pan infamant, voire inavouable, de l'histoire d'une musique dont on préfère généralement exalter le militantisme politique ou la conscience sociale et raciale. Un très grand nombre de travaux sur le sujet, même s'ils ne l'escamotent pas, l'éclipsent généralement en explorant des aspects esthétiques et contestataires qui légitiment cette musique au détriment d'un aspect sociologique et criminel notable.

Or, les simplifications auxquelles cette musique a régulièrement donné lieu sont symptomatiques, comme la réaction de Pinkney après la publication du livre de Wilson, d'un rapport non neutre à un objet. Elles révèlent un positionnement idéologique qui atténue considérablement la valeur analytique du travail des spécialistes qui les ont formulées et viennent, sous le couvert de l'analyse, renforcer un discours partisan dont nous avons vu qu'il n'est guère compatible avec une démarche scientifique qui implique la neutralisation de l' objet d'étude par un effort de distanciation et qui exige d'adopter une approche méthodologique dont l'absence de partialité est susceptible d'apporter, comme Wilson le fit avec The Declining Significance of Race, une mise en perspective bien plus pertinente. 


\section{BIBLIOGRAPHIE}

Bachelard, Gaston, La philosophie du non : Essai d'une philosophie du nouvel esprit scientifique (Paris : PUF, 2005)

Bourdieu, Pierre avec Jean-Claude Chamboredon et Jean-Claude Passeron, Le Métier de Sociologue (Paris : Editions de l'EHESS, 1968)

Gates Jr., Henry Louis \& William J. Wilson, « The Two Nations of Black America » http:// www.pbs.org/wgbh/pages/frontline/shows/race/interviews/wilson.html (consulté le 7/10/ 2008)

Henderson, Vivian W., "Economic Dimensions in Race Relations", in Masuoka, Jitsuichi, Valien, Preston, Race Relations: Problems and Theory (Ayer Co Pub, 1975), pp-252-266

Pinkney, Alfonso, The Myth of Black Progress (Cambridge: Cambridge University Press, 1984)

Wilson, William Julius, The Declining Significance of Race: blacks and changing American institutions $2^{\text {nd }}$ edition (Chicago: The University of Chicago Press, 1980)

(b), "The Black Community: Race and Class" in Ronald Takaki (ed.) From Different Shores:Perspectives on Race and Ethnicity in America(second edition) (New York: Oxford University Press, 1994), pp. 243-250.

Weber, Max, Le Savant et le Politique (Paris : Plon, 1991)

\section{NOTES}

1. (Weber, 23)

2. Cet entretien, intitulé «The Two Nations of Black America » est retranscrit en intégralité sur le site de la chaîne à l'adresse: http://www.pbs.org/wgbh/pages/frontline/shows/race/ interviews/wilson.html

3. William Julius Wilson rajouta d'ailleurs un chapitre supplémentaire dans la seconde édition de The Declining Significance of Race intitulé « Race, Class and Public Policy » dans lequel il revient sur les commentaires et ouvrages qui suivirent la première parution de son livre (pp. 155-82).

4. "But criticism is nothing new for the 60-year-old sociologist. He first caused an uproar in 1978 with his landmark claim in The Declining Significance of Race that class and economics play a more relevant role than race in the plight of the black urban poor." (http://www.motherjones.com/ news/qa/1996/09/early.html)

5. Wilson est notamment mis dans le même panier que Daniel Moynihan, qui fut conseiller de Richard Nixon et l'auteur d'un rapport polémique intitulé The Negro Family: The Case for National Action qui donna lieu à de nombreuses réactions. Un grand nombre de réactions suscitées par ce rapport dans le monde universitaire et politique furent répertoriées par Lee Rainwater et William Lancey dans un ouvrage intitule The Moynihan Report and the Politics of Controversy (Cambridge, Mass. : MIT Press, 1967). Pour une analyse plus récente, lire Dean E. Robinson "The Black Family and US Social Policy: Moynihan's Unintended Legacy? " in Revue Française d'Etudes Américaines, $\mathrm{n}^{\circ} 97$-2003/3, pp.118-128.

6. Son entreprise s'apparente ainsi à celle de Robert B. Hill dont le livre The Illusion of Black Progress, auquel Wilson répond d'ailleurs dans la postface de l'édition de 1980, rejetait déjà plusieurs arguments de The Declining Significance of Race.

7. (Pinkney, 13) 
8. (Pinkney 13)

9. (Pinkney, 14-15)

10. "Perhaps the most controversial and disturbing report is that by William Wilson." (Pinkney, 82). William Julius Wilson revient d'ailleurs sur l'ampleur et la nature des polémiques suscitées par la publication de The declining Significance of Race, non seulement dans le monde universitaire mais également dans les media, dans la deuxième édition de son ouvrage (Wilson, 155).

11. Des divergences sur lesquelles Wilson s'explique de nouveau dans cette deuxième édition. Il explique par exemple certaines différences d'interprétations des mêmes données soit par une prise en compte de variables socio-économiques occultées par ses détracteurs, soit par une variation des échantillons statistiques. Loin d'être anecdotiques, ces batailles statistiques mettent à jour des divergences méthodologiques profondes entre le travail de Wilson et celui de ces détracteurs. William Julius Wilson met notamment en évidence les lacunes méthodologiques du rapport préparé par Robert B. Hill pour la National Urban League, sur lequel Pinkney s'appuie pour invalider sa thèse, et démontre qu'une prise en compte du revenu imposable (aggregate income) pour analyser comparativement l'évolution du fossé entre les classes défavorisées et moyennes dans les communautés noires et blanches s'avère bien plus pertinente et révélatrice que l'analyse de données moins poussée effectuée par Hill. (Wilson, 172)

12. Wilson revient longuement sur sa démarche dans l'entretien accordé à Henry Louis Gates Jr. mentionné en début d'article (note 2).

13. voir Vivian W. Henderson, "Economic Dimensions in Race Relations", dans Masuoka, Jitsuichi, Valien, Preston, Race Relations: Problems and Theory (Ayer Co Pub, 1975), pp. 252-266. Par ailleurs, Wilson, dans le chapitre 8 de l'édition de 1980, rappelle, s'appuyant sur les recherches de Lewis Killian, qu'il n'était ni le premier à affirmer que les problèmes économiques des Noirs ne tenaient pas exclusivement au facteur racial, et que des membres éminents de la NAACP avaient tenu des propos convergents bien avant lui. (Wilson, pp.162-64)

14. Ce que Wilson précise d'ailleurs à Gates en ces termes: "For example, the late black economist, Vivian Henderson, stated years ago just before his death that it's as if racism having put blacks in their economic place stepped aside to see changes in technology and changes in the economy destroy that place."

15. (Wilson b., 244)

16. En 1978, après avoir vu sa demande d'admission à la faculté de médecine de l'Université de Californie à Davis refusée à deux reprises en dépit de résultats supérieurs à ceux de candidats issus de minorités, Alan Bakke décida de porter plainte devant les tribunaux. Cet étudiant estimait être victime de la politique de discrimination positive (affirmative action) en vigueur dans certaines universités californiennes sous la forme d'une discrimination inversée (reverse discrimination). L'affaire arrivera jusqu'à la Cour Suprême qui lui donnera raison et jugera le programme d'admission illégal. Si la Cour établissait la validité constitutionnelle des mesures de discrimination positive, elle les limitait néanmoins afin d'éviter qu'elles ne réduisent pas les chances d'accès aux candidats issus de la majorité.

Wilson regrette en effet le fait que cette affaire retentissante ait éclipsé le passage du Humphry/ Hawkins full employement bill (1978), un programme ambitieux de mesures gouvernementales visant à réduire le taux chômage à moins de $4 \%$ et à stimuler l'emploi dans le privé. Selon lui, le problème de dislocation économique qui affectait les Noirs les plus pauvres constituait un enjeu bien plus crucial que celui de la discrimination raciale. (Wilson, 182)

17. (Weber, 8)

18. Comme cela fut par exemple le cas pour Wilson lorsqu'il devint conseiller du Président Clinton

19. (Pinkney, 17)

20. Cette démarche sociologique est présentée en détail dans Le Métier de Sociologue où les auteurs expliquent que la base de toute méthode scientifique est d'écarter systématiquement les 
prénotions, et de substituer une méthode objective à des ressentis ou à des impressions personnelles.

21. "Ils ne sont plus des socs de charrue pour ameublir l'immense champ de la pensée contemplative, mais des glaives pour attaquer des adversaires, bref, des moyens de combat. » (Weber, 80)

22. (Pinkney p.ix)

23. Des conditions par exemple énoncées par Gaston Bachelard dans La philosophie du non.

24. Nation Conscious Rap, une série d'entretiens avec plusieurs figures emblématiques du début du mouvement

hip hop éditée par Joseph D. Eure et James E. Spady présente un exemple patent du type de contradictions qui ressortent régulièrement des écrits sur la musique rap. Par ailleurs, sans sousévaluer leur très grande valeur informative, un grand nombre de publications sur le rap se distinguent par leur manque de distance avec leur objet d'étude. Dans Buppies, B-boys, Baps and Bohos, Nelson George désigne par exemple les rappeurs par le terme 'brothers' (p.86), un terme qu'utilise également Murray Forman à l'encontre d'un jeune Noir dont il parle dans son ouvrage (p.253) Nelson George, Buppies, B-boys,Baps and Bohos, (New York: Harper Collins, 1992) ; Murray Forman, The 'Hood Comes First: Race, Space and Place in Rap and Hip hop (Middletown, Conn.: Wesleyan University Press, 2003).

\section{RÉSUMÉS}

In this article, I examine some of the reactions that William Julius Wilson's The Declining Significance of Race has triggered among his colleagues and among black activists. I explain that some criticisms, clearly at odds with scientific research, are the result of ideological interpretations that completely divorce Wilson's work from its scholarly context. Through my analysis of the controversy caused by Wilson thesis, I importantly address issues of methodology, analytical rigor, and objectivity in scholarly works and emphasize highly biased assumptions presented by myopic researchers who let racial and political ideology take precedence over objectivity.

\section{AUTEUR}

\section{DAVID DIALLO}

David Diallo est maître de conférences en études anglophones à l'Université MontesquieuBordeaux 4. Ses domaines de recherche incluent la production culturelle des ghettos noirs et les pratiques criminelles qui y ont cours. http://www.u-bordeaux4.fr/accueil/recherche/ enseignants-chercheurs/chercheur/?contentId $=40120$ 